PSFC/JA-05-25

\title{
A New, Explicitly Collisional Contribution to the Gyroviscosity and the Radial Electric Field in a Collisional Tokamak
}

\author{
Catto $^{1}$, Peter J. and Simakov ${ }^{2}$, Andrei N. \\ ${ }^{1}$ MIT Plasma Science and Fusion Center, Cambridge, MA 02139 USA \\ ${ }^{2}$ Los Alamos National Laboratory, Los Alamos, NM 87545, USA
}

This work was supported by the U.S. Department of Energy, Grant No. DE-FG02-91ER-54109.

Submitted to Physics of Plasmas, September 2005 


\title{
A New, Explicitly Collisional Contribution to the Gyroviscosity and the Radial Electric Field in a Collisional Tokamak
}

\author{
$\underline{\text { P. J. Catto }}^{1}$ and A. N. Simakov ${ }^{2}$

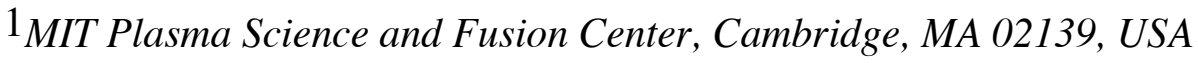 \\ 2 Los Alamos National Laboratory, Los Alamos, NM 87545, USA
}

\begin{abstract}
An additional contribution to the ion viscosity for a collisional plasma is evaluated and found to be the same order as other temperature gradient terms in the collisional perpendicular viscosity. The new contribution arises because of an explicitly collisional portion of the ion distribution function. The evaluation of the Pfirsch-Schlüter radial electric field in a collisional tokamak of arbitrary cross section is extended to retain the new contribution. In a spherical tokamak this new contribution must be retained in determining the radial electric field, while in a conventional tokamak it is small by $1 / \mathrm{q}^{2}$, where $\mathrm{q}$ is the safety factor.
\end{abstract}

\section{Introduction}

The gyroviscosity and perpendicular collisional viscosity in a strongly magnetized plasma are normally evaluated by using various moments of the kinetic equation $[1,2]$. In doing so the gyrophase dependent portion of the ion distribution function that contains a term proportional to the product of gyroradius and the ion collision frequency is assumed negligible. However, we find that this explicitly collisional piece results in a collisional heat flux contribution to the gyroviscosity that is formally the same order as other temperature gradient terms in the collisional perpendicular viscosity. By evaluating the new explicitly collisional gyroviscous term we obtain the full expression for the viscosity for a collisional plasma for the first time. Interestingly, its retention simply replaces the lowest order (diamagnetic plus collisional parallel) heat flow terms in the gyroviscosity by the full heat flow including the classical collisional cross field heat flux.

In addition, the new term in the gyroviscosity allows us to complete the description of a collisional tokamak by evaluating its contribution to the radial electric field. Only for a spherical tokamak can this new term become significant.

\section{Collisional Portion of the Gyrophase Dependent Ion Distribution Function}

In Ref. [2], a $\vee / \Omega$ correction to the gyroviscosity that is formally the same order as the perpendicular viscosity, was not retained, where $v$ and $\Omega=\mathrm{eB} / \mathrm{Mc}$ are the ion-ion 
collision and gyro-frequencies. This collisional term was neglected in the ion distribution function $\mathrm{f}$ because it was only expected to contribute to the collisional ion heat flux that is most easily evaluated by a moment approach. If we order $\nu / \Omega \sim \rho / \mathrm{L} \equiv \delta$, with $\rho$ the ion gyroradius and $\mathrm{L}$ the perpendicular scale length, then the new term is an order $\delta^{3}$ correction to the isotropic pressure. To see how it arises in a collisional plasma we follow Ref. [2] by introducing the shifted velocity variable $\vec{w}=\vec{V}-\vec{V}$ and employing ion momentum conservation to obtain the following form of the kinetic equation:

$\Omega \overrightarrow{\mathrm{w}} \times \overrightarrow{\mathrm{n}} \cdot \nabla_{\mathrm{w}} \mathrm{f}+\mathrm{w} \cdot \nabla \mathrm{f}+(\mathrm{Mn})^{-1}(\nabla \mathrm{p}+\nabla \cdot \overrightarrow{\mathrm{n}}) \cdot \nabla_{\mathrm{w}} \mathrm{f}+\frac{\partial \mathrm{f}}{\partial \mathrm{t}}+\overrightarrow{\mathrm{V}} \cdot \nabla \mathrm{f}-\overrightarrow{\mathrm{w}} \cdot \nabla \overrightarrow{\mathrm{V}} \cdot \nabla \mathrm{f}=\mathrm{C}+\mathrm{C}_{\mathrm{ei}}$

where $M, n=\int d^{3} w f, p=n T=(M / 3) \int d^{3} w w^{2} f$, and $\vec{V}=n^{-1} \int d^{3} \overrightarrow{v v f}$ are the ion mass, density, pressure, and mean velocity; $\mathrm{C}$ and $\mathrm{C}_{\mathrm{ei}}$ are the ion-ion and ion-electron collision operators; and $\vec{n}=\vec{B} / B$. The ion viscosity $\vec{\pi}$ is defined by $\vec{\pi}=M \int d^{3} w\left(\vec{w} \vec{w}-\vec{I} w^{2} / 3\right) f$, where $\overrightarrow{\mathrm{I}}$ is the unit dyad. Expanding order by order in $\delta$ by writing $f=f_{0}+f_{1}+f_{2}+\ldots$, we find as usual that

and

$$
\mathrm{f}_{0}=\mathrm{n}(\mathrm{M} / 2 \pi \mathrm{T})^{1 / 2} \exp \left(-\mathrm{Mw}^{2} / 2 \mathrm{~T}\right)
$$

$$
\mathrm{f}_{1}=-\frac{2 \mathrm{Mf}_{0}}{5 \mathrm{pT}}\left[\overrightarrow{\mathrm{q}} \cdot \overrightarrow{\mathrm{w}} \mathrm{L}_{1}^{(3 / 2)}\left(\mathrm{x}^{2}\right)-\frac{4}{15} \mathrm{q}_{\|} \mathrm{w}_{\|} \mathrm{L}_{2}^{(3 / 2)}\left(\mathrm{x}^{2}\right)\right],
$$

where $\mathrm{x}^{2}=\mathrm{Mw}^{2} / 2 \mathrm{~T}, \mathrm{~L}_{\mathrm{j}}^{(\mathrm{K})}\left(\mathrm{x}^{2}\right)$ are the generalized Laguerre polynomials with $\mathrm{L}_{1}^{(3 / 2)}\left(\mathrm{x}^{2}\right)=5 / 2-\mathrm{x}^{2}$ and $\mathrm{L}_{2}^{(3 / 2)}\left(\mathrm{x}^{2}\right)=\left[35 / 4-7 \mathrm{x}^{2}+\mathrm{x}^{4}\right] / 2$, and here $\overrightarrow{\mathrm{q}}$ is only the lowest order ion heat flux $\overrightarrow{\mathrm{q}}=(5 \mathrm{p} / 2 \mathrm{M} \Omega) \overrightarrow{\mathrm{n}} \times \nabla \mathrm{T}+\mathrm{q}_{\mid \|} \overrightarrow{\mathrm{n}}$ with $\mathrm{q}_{\|}=-(125 \mathrm{p} / 32 \mathrm{M} v) \overrightarrow{\mathrm{n}} \cdot \nabla \mathrm{T}$ and $v$ is defined as $v=4 \pi^{1 / 2} \mathrm{ne}^{4} \ln \Lambda / 3 \mathrm{M}^{1 / 2} \mathrm{~T}^{3 / 2}$.

The new term arises in next order from the solution of

$$
\Omega \overrightarrow{\mathrm{w}} \times \overrightarrow{\mathrm{n}} \cdot \nabla_{\mathrm{w}} \mathrm{f}_{\mathrm{c}}=\mathrm{C}_{1}\left\{\tilde{\mathrm{f}}_{1}\right\},
$$

where $\tilde{f}_{1}$ is the gyrophase dependent portion of $\mathrm{f}_{1}, \mathrm{f}_{\mathrm{c}} \sim \delta^{2} \mathrm{f}_{0}$, and $\mathrm{C}_{1}$ is the linearized ionion collision operator. Only the $(2 \mathrm{M} / 5 \mathrm{pT}) \overrightarrow{\mathrm{q}} \cdot \overrightarrow{\mathrm{w}}_{\perp} \mathrm{x}^{2} \mathrm{f}_{0}=\Omega^{-1} \mathrm{f}_{0} \mathrm{x}^{2} \overrightarrow{\mathrm{w}}_{\perp} \cdot \overrightarrow{\mathrm{n}} \times \nabla \ell \mathrm{nT}$ part of $\tilde{\mathrm{f}}_{1}$ does not vanish when operated on by $\mathrm{C}_{1}$. Then, using the result from Appendix $\mathrm{C}$ of [3] gives the explicitly collisional portion of the order $\delta^{2}$ solution not retained elsewhere [2, 4] to be the gyrophase dependent piece

where

$$
\tilde{\mathrm{f}}_{\mathrm{c}}=\frac{v \mathrm{Q}(\mathrm{x}) \mathrm{f}_{0}}{\Omega^{2}} \overrightarrow{\mathrm{w}}_{\perp} \cdot \nabla \ell \mathrm{nT},
$$

$$
Q=-\frac{3(2 \pi)^{1 / 2}}{x}\left[\left(1-\frac{5}{2 x^{2}}\right) E(x)+\frac{5}{2 x^{2}} E^{\prime}(x)\right],
$$


with $E(x)=2 \pi^{-1 / 2} \int_{0}^{x} d t \exp \left(-t^{2}\right)$ the error function and $E^{\prime}(x)$ its derivative. When used in the moment description to evaluate the viscosity this term leads to the new contribution ignored in all previous work.

\section{New Explicitly Collisional Contribution to the Gyroviscosity}

Retaining the explicitly collisional and gyrophase dependent contribution from Eq. (5) the full ion viscosity becomes

$$
\vec{\pi}=\mathrm{M} \int \mathrm{d}^{3} \mathrm{wf}\left(\overrightarrow{\mathrm{w}} \overrightarrow{\mathrm{w}}-\overrightarrow{\mathrm{I}}^{2} / 3\right)=\vec{\pi}_{\|}+\vec{\pi}_{\mathrm{g}}+\vec{\pi}_{\perp}+\vec{\pi}_{\mathrm{c}},
$$

where $\vec{\pi}_{\|}$and $\vec{\pi}_{\perp}$ are the parallel and perpendicular collisional viscosities and $\vec{\pi}_{\mathrm{g}}$ is the gyroviscosity. The remaining term $\vec{\pi}_{\mathrm{c}}$ is the collisional viscosity due to $\tilde{\mathrm{f}}_{\mathrm{c}}$ and it must be determined by solving

$$
\Omega\left(\vec{\pi}_{c} \times \vec{n}-\vec{n} \times \vec{\pi}_{c}\right)=\vec{K}_{c}
$$

where

$$
\overrightarrow{\mathrm{K}}_{\mathrm{c}} \equiv \nabla \cdot\left[\mathrm{M} \int \mathrm{d}^{3} \mathrm{w}_{\mathrm{c}} \tilde{\mathrm{f}}_{\mathrm{w}} \overrightarrow{\mathrm{w}}\left(\overrightarrow{\mathrm{w}} \overrightarrow{\mathrm{w}}-\overrightarrow{\mathrm{I}}_{\mathrm{w}} 2 / 3\right)\right]+(\overrightarrow{\mathrm{I}}-3 \overrightarrow{\mathrm{n}} \overrightarrow{\mathrm{n}}) \overrightarrow{\mathrm{n}} \cdot\left\{\nabla \cdot\left[(\mathrm{M} / 2) \int \mathrm{d}^{3} \mathrm{wf}_{\mathrm{c}} \overrightarrow{\mathrm{w}}\left(\overrightarrow{\mathrm{w}} \overrightarrow{\mathrm{w}}-\overrightarrow{\mathrm{I}}_{\mathrm{w}} 2 / 3\right)\right]\right\} \cdot \overrightarrow{\mathrm{n}}
$$

Notice that $\vec{K}_{c}$ is traceless with $\vec{n} \cdot \vec{K}_{c} \cdot \vec{n}=0$ as required. Moreover, the collisional term $\tilde{f}_{\mathrm{c}}$ contributes to the viscosity $\vec{\pi}$ through a term similar to the one that leads to the gyroviscosity $\vec{\pi}_{\mathrm{g}}$ through order $\delta^{2}$ [2], since the replacements $\tilde{\mathrm{f}}_{\mathrm{c}} \rightarrow \mathrm{f}_{1}$ and $\overrightarrow{\mathrm{K}}_{\mathrm{c}} \rightarrow \overrightarrow{\mathrm{K}}_{\mathrm{g}}$ in Eq. (9) give the usual gyroviscosity [2, 4, 5] as the solution of $\Omega\left(\vec{\pi}_{g} \times \vec{n}-\vec{n} \times \vec{\pi}_{g}\right)=\vec{K}_{g}$. The only order $\delta^{3}$ correction to the gyroviscous result is collisional and comes from $\tilde{\mathrm{f}}_{\mathrm{c}}$ since all other order $\delta^{2}$ corrections to $f_{0}$ were found in [2] to be even functions of $\vec{w}$ and therefore unable to contribute to $\overrightarrow{\mathrm{K}}_{\mathrm{c}}$.

Next, we evaluate the integrals in $\overrightarrow{\mathrm{K}}_{\mathrm{c}}$ as in [2] to find

$$
\overrightarrow{\mathrm{K}}_{\mathrm{c}}=\frac{2}{5}\left[\nabla \overrightarrow{\mathrm{q}}_{\mathrm{c}}+\left(\nabla \overrightarrow{\mathrm{q}}_{\mathrm{c}}\right)^{\mathrm{T}}-\frac{2}{3} \overrightarrow{\mathrm{I}} \nabla \cdot \overrightarrow{\mathrm{q}}_{\mathrm{c}}\right]+\frac{1}{5}(\overrightarrow{\mathrm{I}}-3 \overrightarrow{\mathrm{n}} \overrightarrow{\mathrm{n}}) \overrightarrow{\mathrm{n}} \cdot\left[\nabla \overrightarrow{\mathrm{q}}_{\mathrm{c}}+\left(\nabla \overrightarrow{\mathrm{q}}_{\mathrm{c}}\right)^{\mathrm{T}}-\frac{2}{3} \overrightarrow{\mathrm{I}} \nabla \cdot \overrightarrow{\mathrm{q}}_{\mathrm{c}}\right] \cdot \overrightarrow{\mathrm{n}},
$$

where $\overrightarrow{\mathrm{q}}_{\mathrm{c}}$ is the usual classical perpendicular ion heat flux

$$
\overrightarrow{\mathrm{q}}_{\mathrm{c}}=-\left(2 \mathrm{p} v / \mathrm{M} \Omega^{2}\right) \nabla_{\perp} \mathrm{T} .
$$

The solution to Eq. (8) for $\vec{\pi}_{\mathrm{c}}$ is $[1,2]$

$$
\vec{\pi}_{c}=(1 / 4 \Omega)\left[\vec{n} \times \vec{K}_{c} \cdot(\vec{I}+3 \vec{n} \vec{n})-(\vec{I}+3 \vec{n} \vec{n}) \cdot \vec{K}_{c} \times \vec{n}\right]
$$

or

$$
\vec{\pi}_{c}=(1 / 10 \Omega)\left\{\vec{n} \times\left[\nabla \overrightarrow{\mathrm{q}}_{c}+\left(\nabla \overrightarrow{\mathrm{q}}_{\mathrm{c}}\right)^{\mathrm{T}}\right] \cdot(\overrightarrow{\mathrm{I}}+3 \overrightarrow{\mathrm{n}} \overrightarrow{\mathrm{n}})-(\overrightarrow{\mathrm{I}}+3 \overrightarrow{\mathrm{n}} \overrightarrow{\mathrm{n}}) \cdot\left[\nabla \overrightarrow{\mathrm{q}}_{\mathrm{c}}+\left(\nabla \overrightarrow{\mathrm{q}}_{\mathrm{c}}\right)^{\mathrm{T}}\right] \times \overrightarrow{\mathrm{n}}\right\}
$$

which is of the same form as the gyroviscosity. As a result, we can add $\vec{\pi}_{\mathrm{g}}$ and $\vec{\pi}_{\mathrm{c}}$ together to get the compact form

$$
\begin{gathered}
\vec{\pi}_{\mathrm{g}}+\vec{\pi}_{\mathrm{c}}=\frac{1}{4 \Omega}\left\{\overrightarrow{\mathrm{n}} \times\left[\mathrm{p} \nabla \overrightarrow{\mathrm{V}}+\frac{2}{5} \nabla \overrightarrow{\mathrm{q}}+\left(\mathrm{p} \nabla \overrightarrow{\mathrm{V}}+\frac{2}{5} \nabla \overrightarrow{\mathrm{q}}\right)^{\mathrm{T}}\right] \cdot(\overrightarrow{\mathrm{I}}+3 \overrightarrow{\mathrm{n}} \overrightarrow{\mathrm{n}})\right. \\
\left.-(\overrightarrow{\mathrm{I}}+3 \overrightarrow{\mathrm{n}} \overrightarrow{\mathrm{n}}) \cdot\left[\mathrm{p} \nabla \overrightarrow{\mathrm{V}}+\frac{2}{5} \nabla \overrightarrow{\mathrm{q}}+\left(\mathrm{p} \nabla \overrightarrow{\mathrm{V}}+\frac{2}{5} \nabla \overrightarrow{\mathrm{q}}\right)^{\mathrm{T}}\right] \times \overrightarrow{\mathrm{n}}\right\}
\end{gathered}
$$


where $\overrightarrow{\mathrm{q}}$ is the total ion heat flux

$$
\overrightarrow{\mathrm{q}}=(5 \mathrm{p} / 2 \mathrm{M} \Omega) \overrightarrow{\mathrm{n}} \times \nabla \mathrm{T}-(125 \mathrm{p} / 32 \mathrm{M} v) \overrightarrow{\mathrm{n}} \overrightarrow{\mathrm{n}} \cdot \nabla \mathrm{T}-\left(2 \mathrm{p} v / \mathrm{M} \Omega^{2}\right) \nabla_{\perp} \mathrm{T} .
$$

Consequently, the generalized gyroviscosity $\vec{\pi}_{g}+\vec{\pi}_{c}$ is most conveniently viewed as having an explicitly collisional modification. Earlier results $[2,4,5]$ appear to be of the same form; however, only the diamagnetic and parallel heat fluxes [the first two terms of Eq. (15), respectively] were retained. It is important to realize that the full expression for the gyroviscosity cannot be obtained from standard drift kinetics [6], but rather must be obtained by extending drift kinetics to retain all order $\delta^{2}$ corrections [7].

\section{Collisional Perpendicular Viscosity}

The full expressions for the collisional perpendicular viscosity $\vec{\pi}_{\perp}=\vec{\pi}_{\perp 1}+\vec{\pi}_{\perp 2}$ are rather involved [2], but in a streamlined form they may be written as

$$
\vec{\pi}_{\perp 1}=-\frac{3 v}{10 \Omega^{2}}[\overrightarrow{\mathrm{W}}+3 \overrightarrow{\mathrm{n}} \overrightarrow{\mathrm{n}} \cdot \overrightarrow{\mathrm{W}}+3 \overrightarrow{\mathrm{W}} \cdot \overrightarrow{\mathrm{n}} \overrightarrow{\mathrm{n}}+(1 / 2)(\overrightarrow{\mathrm{I}}-15 \overrightarrow{\mathrm{n}} \overrightarrow{\mathrm{n}})(\overrightarrow{\mathrm{n}} \cdot \overrightarrow{\mathrm{W}} \cdot \overrightarrow{\mathrm{n}})-(1 / 2)(\overrightarrow{\mathrm{I}}-\overrightarrow{\mathrm{n}} \overrightarrow{\mathrm{n}})(\overrightarrow{\mathrm{W}}: \overrightarrow{\mathrm{I}})]
$$

and

$$
\vec{\pi}_{\perp 2}=-\frac{9 \mathrm{M} v}{200 \mathrm{pT} \Omega}\left[\left(\overrightarrow{\mathrm{q}}+\frac{31}{15} \overrightarrow{\mathrm{q}}_{\|}\right)(\overrightarrow{\mathrm{n}} \times \overrightarrow{\mathrm{q}})+(\overrightarrow{\mathrm{n}} \times \overrightarrow{\mathrm{q}})\left(\overrightarrow{\mathrm{q}}+\frac{31}{15} \overrightarrow{\mathrm{q}}_{\|}\right)\right]
$$

with

$$
\begin{aligned}
\overrightarrow{\mathrm{W}} \equiv \mathrm{p} \nabla \overrightarrow{\mathrm{V}}+\frac{2}{5} \nabla \overrightarrow{\mathrm{q}}-\frac{3}{10 \mathrm{p}}(\mathrm{p} \nabla \overrightarrow{\mathrm{q}}-\overrightarrow{\mathrm{q}} \nabla \mathrm{p}) & -\frac{1}{100 \mathrm{p}}\left(3 \mathrm{p} \nabla \overrightarrow{\mathrm{q}}_{||}+5 \overrightarrow{\mathrm{q}}_{||} \nabla \mathrm{p}\right)-\frac{1}{400 \mathrm{~T}}\left(90 \overrightarrow{\mathrm{q}}-13 \overrightarrow{\mathrm{q}}_{||}\right) \nabla \mathrm{T} \\
& + \text { Transpose } .
\end{aligned}
$$

In Eqs. (17) and (18) only the lowest order $\vec{q}$ is required [the first two terms of Eq. (15)]. Comparing Eqs. (14) - (15) with (16) - (18) we see that the contribution to the gyroviscosity from the perpendicular collisional ion heat flux is the same order as the perpendicular collisional viscosity, that is, $v / \Omega$ times smaller than the lowest order gyroviscosity. We remark here that Eqs. (16) - (18) are the full expressions for the collisional perpendicular viscosity since the terms proportional to $\vec{I}$ and $\vec{n} \vec{n}$ are retained.

\section{Radial Electric Field in a Tokamak}

To determine the Pfirsch-Schlüter radial electric field in a collisional tokamak of arbitrary cross section the radial flux of toroidal angular momentum $\left\langle\mathrm{R}^{2} \nabla \xi \cdot \vec{\pi} \cdot \nabla \psi\right\rangle$ must be evaluated, where $\langle\ldots\rangle$ denotes a flux surface average. This flux was evaluated in [8] except for the contribution due to the new explicitly collisional term $\vec{\pi}_{c}$, which is most easily evaluated by dotting Eq. (8) from both sides by $\mathrm{R}^{2} \nabla \xi$, where $\mathrm{R}$ is the major radius and $\zeta$ is the toroidal angle. Taking $\overrightarrow{\mathrm{B}}=\mathrm{I} \nabla \zeta+\nabla \zeta \times \nabla \psi$, with $\mathrm{I}=\mathrm{I}(\psi)$ and $\psi$ the poloidal flux function, we find upon flux surface averaging 


$$
\left\langle\mathrm{R}^{2} \nabla \xi \cdot \vec{\pi}_{\mathrm{c}} \cdot \nabla \psi\right\rangle=(\mathrm{B} / 2 \Omega)\left\langle\mathrm{R}^{4} \nabla \zeta \cdot \overrightarrow{\mathrm{K}}_{\mathrm{c}} \cdot \nabla \zeta\right\rangle .
$$

Evaluating $\mathrm{R}^{4} \nabla \xi \cdot \overrightarrow{\mathrm{K}}_{\mathrm{c}} \cdot \nabla \xi$ from Eq. (10) gives

so that

$$
\mathrm{R}^{4} \nabla \zeta \cdot \overrightarrow{\mathrm{K}}_{\mathrm{c}} \cdot \nabla \zeta=\frac{4}{5} \mathrm{R}^{4} \nabla \zeta \cdot \nabla \overrightarrow{\mathrm{q}}_{\mathrm{c}} \cdot \nabla \zeta-\frac{2}{5}\left(\mathrm{R}^{2}-\frac{\mathrm{I}^{2}}{\mathrm{~B}^{2}}\right) \nabla \cdot \overrightarrow{\mathrm{q}}_{\mathrm{c}}+\frac{2}{5}\left(\mathrm{R}^{2}-\frac{3 \mathrm{I}^{2}}{\mathrm{~B}^{2}}\right) \overrightarrow{\mathrm{n}} \cdot \nabla \overrightarrow{\mathrm{q}}_{\mathrm{c}} \cdot \overrightarrow{\mathrm{n}}
$$

$$
\mathrm{R}^{2} \nabla \zeta \cdot \vec{\pi}_{\mathrm{c}} \cdot \nabla \psi=\frac{\mathrm{B}}{5 \Omega}\left[\overrightarrow{\mathrm{q}}_{\mathrm{c}} \cdot \nabla \mathrm{R}^{2}-\left(\mathrm{R}^{2}-\frac{\mathrm{I}^{2}}{\mathrm{~B}^{2}}\right) \nabla \cdot \overrightarrow{\mathrm{q}}_{\mathrm{c}}-\left(\mathrm{R}^{2}-\frac{3 \mathrm{I}^{2}}{\mathrm{~B}^{2}}\right) \overrightarrow{\mathrm{n}} \cdot \nabla \overrightarrow{\mathrm{n}} \cdot \overrightarrow{\mathrm{q}}_{\mathrm{c}}\right],
$$

where $R^{2} B^{2}-I^{2}=\left(R B_{p}\right)^{2}$ with $B_{p}$ the poloidal magnetic field.

If the preceding new term is combined with the results given by Catto and Simakov [8] the complete expresion for the radial flux of toroidal angular momentum becomes

$$
\left\langle\mathrm{R}^{2} \nabla \xi \cdot \vec{\pi} \cdot \nabla \psi\right\rangle=\left\langle\mathrm{R}^{2} \nabla \zeta \cdot\left(\vec{\pi}_{\mathrm{g}}+\vec{\pi}_{\perp}+\vec{\pi}_{\mathrm{c}}\right) \cdot \nabla \psi\right\rangle,
$$

where the radial electric field or electrostatic potential $\Phi$ in the steady state in the absence of sources or sinks is found from $\left\langle\mathrm{R}^{2} \nabla \xi \cdot \vec{\pi} \cdot \nabla \psi\right\rangle=0$.

In effect the new collisional term leads to the replacement $\left\langle\mathrm{R}^{2} \nabla \xi \cdot \vec{\pi}_{\perp} \cdot \nabla \psi\right\rangle \rightarrow\left\langle\mathrm{R}^{2} \nabla \xi \cdot\left(\vec{\pi}_{\perp}+\vec{\pi}_{\mathrm{c}}\right) \cdot \nabla \psi\right\rangle$. The contribution from $\left\langle\mathrm{R}^{2} \nabla \xi \cdot \vec{\pi}_{\mathrm{c}} \cdot \nabla \psi\right\rangle$ is the same order as other $\mathrm{dT} / \mathrm{d} \psi$ terms in $\left\langle\mathrm{R}^{2} \nabla \xi \cdot \vec{\pi}_{\perp} \cdot \nabla \psi\right\rangle$ for comparable perpendicular and parallel scale lengths, as might be the case in a collisional spherical tokamak. It leads to $\mathrm{dT} / \mathrm{d} \psi$ terms that are $1 / \mathrm{q}^{2}$ smaller than the neoclassical terms from $\left\langle\mathrm{R}^{2} \nabla \zeta \cdot \vec{\pi}_{\mathrm{g}} \cdot \nabla \psi\right\rangle$, and these new terms are negligible for a large aspect ratio tokamak or when perpendicular scale lengths are small compared to the major radius. Here $\mathrm{q}$ is the usual tokamak safety factor. To see this in detail recall that the Pfirsch-Schlüter radial heat flux $\overrightarrow{\mathrm{q}}_{\mathrm{ps}}$ is given by

$$
\overrightarrow{\mathrm{q}}_{\mathrm{ps}} \cdot \nabla \psi=-\left(8 \mathrm{I}^{2} \mathrm{p} v / 5 \mathrm{M} \Omega^{2}\right)\left(1-\mathrm{B}^{2} /\left\langle\mathrm{B}^{2}\right\rangle\right) \mathrm{dT} / \mathrm{d} \psi .
$$

As a result, we obtain the usual result [9-11]

$$
\frac{\overrightarrow{\mathrm{q}}_{\mathrm{ps}} \cdot \nabla \psi}{\overrightarrow{\mathrm{q}}_{\mathrm{c}} \cdot \nabla \psi} \sim\left(1-\mathrm{B}^{2} /\left\langle\mathrm{B}^{2}\right\rangle\right) \mathrm{B}^{2} / \mathrm{B}_{\mathrm{p}}^{2} \sim \mathrm{q}^{2} .
$$

We also remark that to obtain the correct expression for $\left\langle\mathrm{R}^{2} \nabla \xi \cdot \vec{\pi}_{\mathrm{g}} \cdot \nabla \psi\right\rangle$ the full expression for the gyroviscosity is required. Standard drift kinetics [6] ignores diamagnetic particle and heat flow terms in the gyroviscosity [7]. These contributions become comparable to those from the parallel flow terms because, for example, $\nabla \cdot \overrightarrow{\mathrm{V}}_{\perp} \sim \nabla \cdot \overrightarrow{\mathrm{V}}_{\|}$and $\nabla \cdot \overrightarrow{\mathrm{q}}_{\perp} \sim \nabla \cdot \overrightarrow{\mathrm{q}}_{\|}$, even though $\left|\overrightarrow{\mathrm{V}}_{\perp}\right| \sim \overrightarrow{\mathrm{V}}_{\|}|/ \mathrm{q}<<| \overrightarrow{\mathrm{V}}_{\|} \mid$and $\left|\overrightarrow{\mathrm{q}}_{\perp}\right| \sim\left|\overrightarrow{\mathrm{q}}_{\|}\right| / \mathrm{q}<<\left|\overrightarrow{\mathrm{q}}_{\|}\right|$ when $\mathrm{q} \gg>1$.

For a collisional or Pfirsch-Schlüter short mean free path ordering in which the plasma flow is sub-sonic [2], we find that there are two interesting limiting cases [8], since all the terms in the gyroviscosity always turn out to be proportional to $\mathrm{dT} / \mathrm{d} \psi$. The 
first is the simpler case of an extremely up-down asymmetric tokamak (for example, just inside the separatrix of a strongly single null divertor configuration) for which the lowest order gyroviscous contribution does not vanish and must be balanced by the leading order collisional viscous effect to determine the radial electric field. The second case is the more complicated case of an up-down symmetric tokamak for which the lowest order gyroviscosity vanishes and so it must be evaluated to higher order and balanced by the lowest order collisional viscosity to determine the radial electric field. In practice both contributions must be retained, and standard drift kinetics cannot be used to obtain the results $[7,8]$. Neither result is altered by the new term evaluated here as long as $\mathrm{q}^{2}>>1$ or if the perpendicular scale lengths are much smaller than the major radius. However, in a spherical tokamak where $\mathrm{q} \sim 1$ must be allowed, Eq. (20) must be added to Eqs. (48) and (57) of Catto and Simakov [8]. In the absence of momentum sources and sinks, the full short mean free path result (including all Pfirsch-Schlüter effects) for the vanishing of the radial flux of toroidal angular momentum in a tokamak of arbitrary cross section, $\left\langle\mathrm{R}^{2} \nabla \xi \cdot \vec{\pi} \cdot \nabla \psi\right\rangle=0$, then becomes

$$
\begin{gathered}
\frac{3 v \mathrm{pB}}{2 \Omega}\left\langle\frac{\mathrm{R}^{2} \mathrm{~B}_{\mathrm{p}}^{2}}{\mathrm{~B}^{2}}\left(\mathrm{R}^{2}+\frac{3 \mathrm{I}^{2}}{\mathrm{~B}^{2}}\right)\right\rangle \frac{\mathrm{d} \omega}{\mathrm{d} \psi}=\frac{5 \mathrm{p}}{2\left\langle\mathrm{~B}^{2}\right\rangle}\left[\frac{\mathrm{cI}}{\mathrm{e}} \frac{\mathrm{dT}}{\mathrm{d} \psi}-\mathrm{u}\right]\left\langle\mathrm{R}^{2} \overrightarrow{\mathrm{B}} \cdot \nabla \ell \mathrm{nB}\right\rangle \\
+\left\langle\nabla \cdot\left[\left(\mathrm{R}^{2}+\frac{3 \mathrm{I}^{2}}{2 \mathrm{~B}^{2}}\right) \overrightarrow{\mathrm{q}}_{\mathrm{ps}}\right]\right\rangle-\left\langle\mathrm{R}^{2}+\frac{7 \mathrm{I}^{2}}{2 \mathrm{~B}^{2}}\right\rangle\left\langle\nabla \cdot \overrightarrow{\mathrm{q}}_{\mathrm{ps}}\right\rangle-\mathrm{F}(\psi)\left\langle\left(\mathrm{R}^{2}-\frac{3 \mathrm{I}^{2}}{2 \mathrm{~B}^{2}}\right)\left(1-\frac{\mathrm{B}^{2}}{\left\langle\mathrm{~B}^{2}\right\rangle}\right)\right\rangle \\
+\left\langle\frac{\mathrm{R}^{2}}{2 \mathrm{~B}^{2}} \frac{\partial \mathrm{B}^{2}}{\partial \psi} \overrightarrow{\mathrm{q}}_{\mathrm{ps}} \cdot \nabla \psi\right\rangle-\frac{1}{\mathrm{I}} \frac{\mathrm{dI}}{\mathrm{d} \psi}\left\langle\left(\mathrm{R}^{2}-\frac{3 \mathrm{I}^{2}}{\mathrm{~B}^{2}}\right) \overrightarrow{\mathrm{q}}_{\mathrm{ps}} \cdot \nabla \psi\right\rangle-4 \pi \frac{\mathrm{d}\left(\mathrm{p}+\mathrm{p}_{\mathrm{e}}\right)}{\mathrm{d} \psi}\left\langle\left(\frac{\mathrm{R}^{2}}{\mathrm{~B}^{2}}-\frac{3 \mathrm{I}^{2}}{\mathrm{~B}^{4}}\right) \overrightarrow{\mathrm{q}}_{\mathrm{ps}} \cdot \nabla \psi\right\rangle \\
+\left\langle\left[\mathrm{R}^{2} \overrightarrow{\mathrm{B}} \cdot \nabla \ell \mathrm{nB}\right]\left[\mathrm{F}(\psi) \int \frac{\mathrm{d} \theta}{\overrightarrow{\mathrm{B}} \cdot \nabla \theta}\left(1-\frac{\mathrm{B}^{2}}{\left\langle\mathrm{~B}^{2}\right\rangle}\right)-\frac{\partial}{\partial \psi} \int \frac{\mathrm{d} \theta \overrightarrow{\mathrm{q}}_{\mathrm{ps}} \cdot \nabla \psi}{\overrightarrow{\mathrm{B}} \cdot \nabla \theta}+\left(\frac{1}{\mathrm{~V}^{\prime}} \int \frac{\mathrm{d} \theta}{\overrightarrow{\mathrm{B}} \cdot \nabla \theta}\right) \frac{\mathrm{d}}{\mathrm{d} \psi}\left(\mathrm{V}^{\prime}\left\langle\overrightarrow{\mathrm{q}}_{\mathrm{ps}} \cdot \nabla \psi\right\rangle\right)\right]\right\rangle \\
+\left\langle\overrightarrow{\mathrm{q}} \cdot \nabla \mathrm{R}^{2}-\left(\mathrm{R}^{2}-\frac{\mathrm{I}^{2}}{\mathrm{~B}^{2}}\right) \nabla \cdot \overrightarrow{\mathrm{q}}_{\mathrm{c}}-\left(\mathrm{R}^{2}-\frac{3 \mathrm{I}^{2}}{\mathrm{~B}^{2}}\right) \overrightarrow{\mathrm{n}} \cdot \nabla \overrightarrow{\mathrm{n}} \cdot \overrightarrow{\mathrm{q}}_{\mathrm{c}}\right\rangle
\end{gathered}
$$

where the only term containing the radial electric field is

$$
\omega=-c\left[\partial \Phi / \partial \psi+(e n)^{-1} \partial \mathrm{p} / \partial \psi\right],
$$

which is a lowest order flux function, and the remaining quantities are

$$
\mathrm{F}=0.7\left[\mathrm{~T}_{\mathrm{e}} /\left(\mathrm{T}_{\mathrm{e}}+\mathrm{T}\right)\right]\left[\left\langle\mathrm{B}^{2}\right\rangle\left\langle\mathrm{B}^{-2}\right\rangle-1\right]^{-1}\left\langle\overrightarrow{\mathrm{q}}_{\mathrm{ps}} \cdot \nabla \psi\right\rangle \mathrm{d} \ell \mathrm{nT} / \mathrm{d} \psi
$$

and

$$
\mathrm{u}=\frac{\mathrm{cI}}{\mathrm{e}\left\langle\mathrm{B}^{2}\right\rangle} \frac{\mathrm{dT}}{\mathrm{d} \psi}\left[1.78+0.057 \frac{\left\langle\mathrm{B}^{2}\right\rangle\left\langle\left(\mathrm{B}^{-2} \overrightarrow{\mathrm{B}} \cdot \nabla \mathrm{B}\right)^{2}\right\rangle}{\left\langle(\overrightarrow{\mathrm{B}} \cdot \nabla \ell \mathrm{nB})^{2}\right\rangle}\right],
$$


with $\overrightarrow{\mathrm{V}}=\omega(\psi) \mathrm{R}^{2} \nabla \zeta+\mathrm{u}(\psi) \overrightarrow{\mathrm{B}}$ to lowest order and $\mathrm{p}_{\mathrm{e}}=\mathrm{nT}_{\mathrm{e}}$ the electron pressure and $\mathrm{p}+\mathrm{p}_{\mathrm{e}}$ the total pressure. The $\theta$ integrals are indefinite and are to be constructed to be periodic and free of secular behavior, while the flux surfaces averages are defined by $\langle\ldots\rangle=\left(\mathrm{V}^{\prime}\right)^{-1} \oint \mathrm{d} \theta(\overrightarrow{\mathrm{B}} \cdot \nabla \theta)^{-1}(\ldots)$ with $\mathrm{V}^{\prime}=\oint \mathrm{d} \theta(\overrightarrow{\mathrm{B}} \cdot \nabla \theta)^{-1}$.

The first term on the right side of (24) only contributes to determining $\omega$ in an up-down asymmmetric tokamak. To become significant it requires extremely strong asymmetry such as might be encountered just inside the separatrix of a strong single null divertor configuration. As a result, we have neglected higher order corrections to it. The second through fourth lines of terms are the Pfirsch-Schlüter contributions to determining $\omega$ and the radial electric field. For large aspect ratio and concentric circular flux surfaces, only the term with the $\mathrm{F}$ coefficient contributes and it leads to the Claassen and $\mathrm{H}$. Gerhauser [12] result:

$$
\left(\mathrm{r} / \Omega_{0}\right) \mathrm{d} \omega / \mathrm{dr} \approx-0.19\left[\mathrm{q}^{3} \rho_{0}^{2} \mathrm{~T}_{\mathrm{e}} / \mathrm{T}^{2}\left(\mathrm{~T}_{\mathrm{e}}+\mathrm{T}\right)\right](\mathrm{dT} / \mathrm{dr})^{2} .
$$

Here the toroidal magnetic field is $\mathrm{B}_{\mathrm{t}}=\mathrm{B}_{0} \mathrm{R}_{0} / \mathrm{R}$, and we define $\Omega_{0}=\mathrm{eB}_{0} / \mathrm{Mc}, \rho_{0}=\mathrm{v}_{\mathrm{i}} / \Omega_{0}$, and $v_{i}=\left(2 T_{i} / M\right)^{1 / 2}$. The fifth line is the new terms evaluated here that are $1 / q^{2}$ smaller than the Pfirsch-Schlüter terms and therefore the same order as the classical terms in the sixth line which are obtained by flux surface averaging Eq. (57) of Catto and Simakov [8] after multiplying it by $(5 \Omega / \mathrm{B})$.

As a final check, we remark that for an isothermal plasma all the terms on the right side of Eq. (24) vanish so that $\omega$ is a constant. In this limit, the solution is consistent with the fact that in the absence of temperature variation a rigidly rotating Maxwellian,

$$
\mathrm{f}_{\mathrm{M}}=\mathrm{n}(\mathrm{M} / 2 \pi \mathrm{T})^{3 / 2} \exp \left[-(\mathrm{M} / 2 \mathrm{~T})\left(\overrightarrow{\mathrm{v}}-\omega \mathrm{R}^{2} \nabla \zeta\right)^{2}\right],
$$

is an exact steady state solution of the ion kinetic equation [3] as long as momentum exchange with the electrons remains weak. It may be written in terms of the energy and canonical angular momentum constants of the motion $E=v^{2} / 2+(e \Phi / M)$ and $\psi_{*}=\psi-(\mathrm{Mc} / \mathrm{e}) \mathrm{R}^{2} \nabla \zeta \cdot \overrightarrow{\mathrm{v}}$ as

$$
\mathrm{f}_{\mathrm{M}}=\mathrm{n}_{0}(\mathrm{M} / 2 \pi \mathrm{T})^{3 / 2} \exp \left[-(\mathrm{ME} / \mathrm{T})-\left(\mathrm{e} \omega \psi_{*} / \mathrm{cT}\right)\right],
$$

with $\mathrm{n}_{0}$ a constant defined by

$$
\mathrm{n}_{0} \equiv \mathrm{n} \exp \left[(\mathrm{e} \Phi / \mathrm{T})+(\mathrm{e} \omega \psi / \mathrm{cT})-\left(\mathrm{M} \omega^{2} \mathrm{R}^{2} / 2 \mathrm{~T}\right)\right]
$$

For constant $\mathrm{T}$ and $\mathrm{n}_{0}$, Eq. (31) gives steady state momentum conservation

$$
M n \omega^{2} R^{2} \nabla \zeta \cdot \nabla\left(R^{2} \nabla \zeta\right)+e n\left[\nabla \Phi-(\omega / c) R^{2} \nabla \xi \times \vec{B}\right]+T \nabla n=0 .
$$

The preceding observation holds regardless of collisionality regime and serves to highlight the importance of ion temperature variation.

Even though edge momentum relaxation is expected to be anomalous, the electric field in the relaxed or steady state need not be anomalous and may be determined at least 
in part by neoclassical considerations. The expressions presented here make it clear that the shear in the electric field and thereby the ion flow is set by the ion temperature profile. This shear and its effect on the ion flow is separate from the sheared axisymmetric zonal flow driven by turbulence. However, the neoclassical contribution to the axisymmetric radial electric field is not normally retained in fluid or kinetic codes.

Interestingly, these neoclassical contributions to the radial electric field and ion flow are sensitive to magnetic topology. If we assume that the plasma current is in the direction of increasing toroidal angle (the $\nabla \zeta$ direction) to make $\psi$ increase outward from the magnetic axis, then the sign of I depends on the toroidal magnetic field direction. As a result, the magnetic topology of a tokamak impacts the radial electric field and ion flow velocity through reversals in the direction of (i) the plasma current or $B_{p}$, (ii) the toroidal magnetic field or I, or (iii) both; or (iv) by changing from lower single null to upper single null operation $(\overrightarrow{\mathrm{B}} \cdot \nabla \rightarrow-\overrightarrow{\mathrm{B}} \cdot \nabla)$.

\section{Conclusions}

For a collisional plasma, the ion viscosity has been shown to be missing an explicitly collisional contribution from the gyroviscosity that is the same order as terms in collisional perpendicular viscosity. This contribution generalizes the standard definition of the gyroviscosity to include the full expression for the ion heat flow (that is, collisional perpendicular as well as diamagnetic and collisional parallel heat flows). The full expressions for these portions of the viscosity are summarized. This new contribution can influence the radial electric field in a tokamak, but does not alter Pfirsch-Schlüter terms since its contribution is the same order as the classical contribution. As a result, it only makes a significant contribution for a spherical tokamak, and is otherwise expected to be small. The full differential equation for the electric field in a tokamak of arbitrary cross section is presented for completeness.

In summary, we have demonstrated that there is an additional contribution to the radial angular momentum flux that arises from an explicitly collisional piece of the gyrophase dependent ion distribution function. The new contribution tends to be small, but is formally the same order as other temperature gradient terms in the perpendicular collisional viscosity and must be retained for spherical tokamaks.

\section{Acknowledgments}

This research was supported by the U.S. Department of Energy Grant No. DEFG02-91ER-54109 at the Plasma Science and Fusion Center of the Massachusetts 
Institute of Technology and Grant No. W-7405-ENG-36 at Los Alamos National Laboratory.

\section{References}

[1] R. D. Hazeltine and J. D. Meiss, Plasma Confinement (Addison-Wesley, Redwood City, CA, 1991), pp. 206-209.

[2] P. J. Catto and A. N. Simakov, Phys. Plasmas 11, 90 (2004).

[3] P. J. Catto, I. Bernstein and M. Tessarotto, Phys. Fluids 30, 2784 (1987).

[4] A. B. Mikhailovskii and V. S. Tsypin, Plasma Phys. 13, 785 (1971) and Beitr. Plasmaphys. 24, 335 (1984).

[5] J. J. Ramos, Phys. Plasmas 12, 052102 (2005).

[6] R. D. Hazeltine, Plasma Phys. 15, 77 (1973).

[7] A. N. Simakov and P. J. Catto, Phys. Plasmas 12, 012105 (2005).

[8] P. J. Catto and A. N. Simakov, Phys. Plasmas 12, 012501 (2005).

[9] R. D. Hazeltine, Phys. Fluids 17, 961 (1974).

[10] F. L. Hinton and R. D. Hazeltine, Rev. Mod. Phys. 48, 239 (1976).

[11] S. P. Hirshman and D. J. Sigmar, Nucl. Fusion 21, 1079 (1981).

[12] H. A. Claassen and H. Gerhauser, Czech. J. Phys. 49, 69 (1999). 